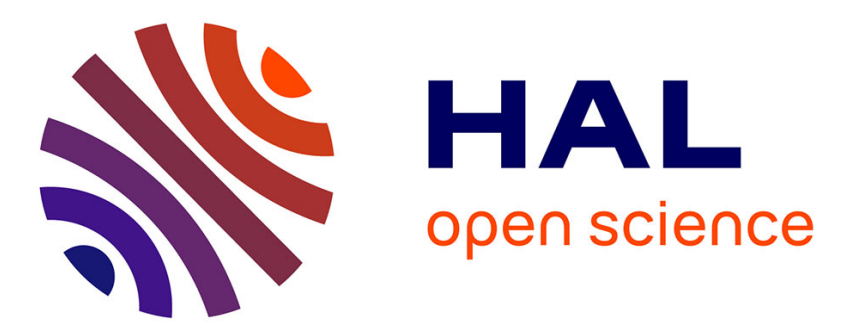

\title{
Orbital moment anisotropy in ultrathin FePt layers
}

Marcio Soares, Anne Lamirand, Aline Y. Ramos, Maurizio de Santis, Hélio C.

N. Tolentino

\section{To cite this version:}

Marcio Soares, Anne Lamirand, Aline Y. Ramos, Maurizio de Santis, Hélio C. N. Tolentino. Orbital moment anisotropy in ultrathin FePt layers. Physical Review B: Condensed Matter and Materials Physics (1998-2015), 2014, 90 (21), pp.214403. 10.1103/PhysRevB.90.214403 . hal-01224306

\section{HAL Id: hal-01224306 https://hal.science/hal-01224306}

Submitted on 4 Nov 2015

HAL is a multi-disciplinary open access archive for the deposit and dissemination of scientific research documents, whether they are published or not. The documents may come from teaching and research institutions in France or abroad, or from public or private research centers.
L'archive ouverte pluridisciplinaire HAL, est destinée au dépôt et à la diffusion de documents scientifiques de niveau recherche, publiés ou non, émanant des établissements d'enseignement et de recherche français ou étrangers, des laboratoires publics ou privés. 


\title{
Orbital moment anisotropy in ultrathin FePt layers
}

\author{
Márcio M. Soares, ${ }^{1}$ Anne D. Lamirand, ${ }^{2,3}$ Aline Y. Ramos,,${ }^{2,3, *}$ Maurizio De Santis, ${ }^{2,3}$ and Hélio C. N. Tolentino ${ }^{2,3,4}$ \\ ${ }^{1}$ European Synchrotron Radiation Facility, 6 Rue Jules Horowitz, BP 220, F-38043 Grenoble Cedex, France \\ ${ }^{2}$ Université Grenoble Alpes, Institut Néel, F-38042 Grenoble, France \\ ${ }^{3}$ CNRS, Institut Néel, F-38042 Grenoble, France \\ ${ }^{4}$ Laboratório Nacional de Luz Síncrotron-LNLS, CP 6192, 13083-970 Campinas, Brazil \\ (Received 10 October 2014; revised manuscript received 2 November 2014; published 1 December 2014)
}

\begin{abstract}
The anisotropy of the Fe orbital moment is investigated in epitaxial ultrathin $(<2 \mathrm{~nm}) \mathrm{FePt}$ layers by angular dependent $\mathrm{X}$-ray magnetic circular dichroism (XMCD) for two distinct epitaxial systems, $\mathrm{CoO} / \mathrm{FePt} / \mathrm{Pt}(001)$ and $\mathrm{Pt} / \mathrm{FePt} / \mathrm{MnPt} / \mathrm{Pt}(001)$. Element selective hysteresis loops obtained from the XMCD signal at the Fe $L_{3}$ edge are used to quantify the anisotropy constants, which are consistent with the corresponding chemical order parameter. A careful procedure for XMCD sum rules data analysis is set up to avoid experimental overestimation of the orbital moment. The measured orbital moment of Fe is found comparable to theoretical values and shows a significant anisotropy. This orbital moment anisotropy is discussed in the light of the current theoretical understanding of magnetocrystalline anisotropy in ordered alloys.
\end{abstract}

DOI: 10.1103/PhysRevB.90.214403

PACS number(s): 75.30.Gw, 75.50.Bb, 75.70.-i, 78.70.Dm

\section{INTRODUCTION}

Magnetic materials presenting large magnetocrystalline anisotropy energy (MAE) with perpendicular magnetization are of great interest in ultrahigh-density magnetic recording and spintronic devices. Systems with perpendicular magnetic anisotropy (PMA) present lower critical current density for magnetization switching by spin-transfer torque in magnetic tunnel junctions [1]. The FePt ordered alloy in the fct $L 1_{0}$ phase, where monoatomic planes of $\mathrm{Pt}$ and $\mathrm{Fe}$ alternate along the $c$ axis, is one of the materials with the largest MAE [2,3]. Combined with a good resistance to corrosion and moderate Curie temperature $\left(T_{C} \sim 750 \mathrm{~K}\right)$ this characteristic makes $L 1_{0} \mathrm{FePt}$ a prime candidate for heat-assisted magnetic recording [2]. The MAE is directly related to the order parameter $S$ of the alloy: The closer it is to a perfect order $(S=1)$, the higher the anisotropy [3-7].

In the ultrathin limit (few nanometers), conventional thermal assisted codeposition leads to poor ordering [4]. Alternate $\mathrm{Fe}$ and $\mathrm{Pt}$ deposition [8] or postannealing of ultrathin $\mathrm{Fe}$ on $\mathrm{Pt}(001)$ [9] are suitable methods for producing ultrathin FePt films with PMA. In such films the order parameter $S$ is only roughly estimated with standard laboratory techniques. Determinations based on surface sensitive techniques such as grazing incidence $\mathrm{x}$-ray diffraction (GIXRD) and proper data analysis procedures are scarce [10]. Moreover, a determination of MAE is still lacking for a few monolayers (ML) thick film.

From a fundamental point of view, the origin of the MAE in $\mathrm{FePt}$ and other transition metal/noble metal alloys has been the subject of intense theoretical research [5-7,11-15]. Even if there is a consensus that the hybridization between the Pt $5 d$ orbitals with a large spin-orbit interaction (SOI) and the highly spin-polarized $\mathrm{Fe} 3 d$ ones is at the origin of the strong MAE in FePt [5,6,11-13], the underlying microscopic process is still under debate. For $3 d$ transition metals, by using perturbation theory, Bruno [16] showed that the MAE should be directly proportional to the orbital moment anisotropy (OMA). Bruno's

\footnotetext{
*Corresponding author: aline.ramos@neel.cnrs.fr
}

theory is developed under the assumption that the exchange splitting is larger than the bandwidth for a $3 d$ element with more than a half-filled $d$ shell. Following this reasoning, it has been calculated for FePt that a dominant contribution to the MAE comes from the $\mathrm{Fe}$ atom [5,15], where the $\mathrm{Fe}$ OMA would be enhanced by hybridization with Pt $5 d$ states. However, this dominant role of OMA in $3 d$ metals was put into question for binary $3 d-5 d$ alloys [17]. According to Soloyev and co-workers [12], the MAE would be entirely related to the Pt sublattice, while the $3 d$ atoms would only act as a source of magnetism by inducing exchange splitting in the Pt atom via orbital hybridization. In this view [6], the anisotropy of the $\mathrm{Pt}$ orbital moment would be the dominant factor.

Experimental determination of the orbital moment $\left(m_{o}\right)$ is commonly obtained from $\mathrm{x}$-ray magnetic circular dichroism (XMCD) sum rules $[18,19]$, but so far reports about angular dependent XMCD measurements in FePt that could provide support to either of these scenarios are still lacking. In addition, there is a serious discrepancy between most experimental determinations of $\mathrm{Fe} m_{o}$ deduced from XMCD sum rules [10,20-22] $\left(0.2-0.3 \mu_{B}\right)$ and those obtained from ab initio calculations $[6,11-13,23]\left(0.06-0.13 \mu_{B}\right)$. The large experimental values for $m_{o}$ reported in the literature can be interpreted as due to the enhancement of the orbital moment in the ordered FePt alloy and the source of MAE [10,22]. Nevertheless, such measurements have been performed for randomly oriented nanoparticles [22] or normally to the films $[20,21]$ and angle dependent measurements are missing.

We report here on a XMCD study of the FePt orbital moment anisotropy performed on two different epitaxial ultrathin systems whose structures (epitaxy, order parameter, surface/interface quality/oxidation) have been previously fully determined by GIXRD [24-26]. For each sample, angular dependent hysteresis loops obtained from the $\mathrm{Fe} L_{3}$ edge XMCD signal allow one to quantify the strong PMA associated with the corresponding chemical order parameter. We propose a procedure for XMCD data analysis limiting systematic errors in the application of sum rules. Using this procedure, we derive the orbital and spin magnetic moments of $\mathrm{Fe}$, in agreement with calculated values. Measurements at normal and grazing 


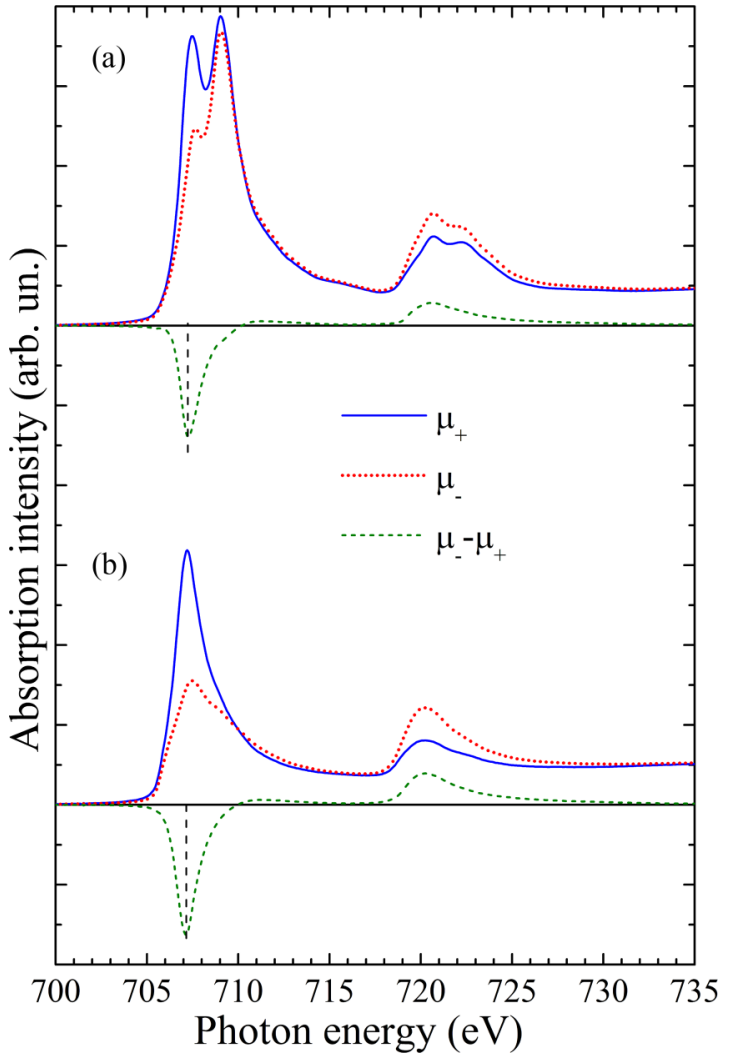

FIG. 1. (Color online) XAS spectra for parallel $\left(\mu_{+}\right)$and antiparallel $\left(\mu_{-}\right)$alignment of the photon helicity with respect to sample magnetization and their difference (XMCD) obtained at $10 \mathrm{~K}$ in normal incidence for (a) sample $\mathrm{A}[\mathrm{CoO}(4.0) / \mathrm{FePt}(1.1) / \mathrm{Pt}(001)]$ and (b) sample $\mathrm{B}[\mathrm{Pt}(1.6) / \mathrm{FePt}(1.6) / \mathrm{MnPt}(3.0) / \mathrm{Pt}(001)]$. The vertical line indicates the photon energy at which hysteresis loops of Fig. 2(b) are measured.

incidence reveal a clear OMA that is discussed in light of the current theoretical understanding of MAE in ordered alloys.

\section{EXPERIMENTAL}

In sample $\mathrm{A}[26]-\mathrm{CoO}(4.0) / \mathrm{FePt}(1.1) / \mathrm{Pt}(001)(\mathrm{nm})$ - the FePt layer is obtained by evaporation of $3 \mathrm{ML}$ of $\mathrm{Fe}$ on a $\mathrm{Pt}(001)$ single crystal at $600 \mathrm{~K}$, followed by $1 \mathrm{ML}$ Pt deposition to limit Fe oxidation. The Fe interdiffusion with the Pt substrate is limited to one atomic plane. The FePt layer is in registry with the substrate $[c / a=0.917(3)]$ and presents $L 1_{0}$ order along the perpendicular $c$ axis with $S=0.7(1)$. Subsequent $\mathrm{CoO}$ reactive growth at $520 \mathrm{~K}$ gives rise to a small interface Fe oxide equivalent to $1 \mathrm{Fe} \mathrm{ML}$, as evidenced by the multiplet oxide signature of the X-ray absorption spectroscopy (XAS) spectra in Fig. 1(a). The XMCD spectrum [green dashed curve in Fig. 1(a)] is characteristic of metallic FePt and is not noticeably affected by this small Fe oxide content. However, a quantitative analysis with XMCD sum rules is limited, as will be discussed later.

Sample $\quad \mathrm{B}-\mathrm{Pt}(1.6) / \mathrm{FePt}(1.6) / \mathrm{MnPt}(3.0) / \mathrm{Pt}(001)$ - is purely metallic and was obtained by alternate layer deposition [24]. The FePt film of 4 bilayers (BL) was grown at $570 \mathrm{~K}$ on an antiferromagnetic $L 1_{0}$ ordered MnPt layer and protected against oxidation by $\mathrm{Pt}$. The FePt (and $\mathrm{MnPt}$ ) layer grows pseudomorphically, with a perpendicular $L 1_{0} c$ axis $[c / a=0.916(2)]$. The FePt/MnPt interface is very sharp and presents a limited chemical roughness $(\approx 0.25 \mathrm{~nm})$. The order parameter $[S=0.5(2)]$ is tainted by a sizable uncertainty due to the superposition of $\mathrm{MnPt}$ and FePt diffraction peaks and a small lateral size of the ordered domains. The absence of Fe oxidation, evidenced by the lack of multiplet structures in the XAS spectra [Fig. 1(b)], enables a proper application of XMCD sum rules. The magnetic properties and exchange coupling of FePt with the corresponding antiferromagnetic layer were described for both sample A [25,27] and sample B [24]. In the case of sample B, the exchange bias field is limited to $8 \mathrm{mT}$ at low temperature and vanishes after training. For sample A, the exchange bias is of $80 \mathrm{mT}$ at low temperature and vanishes above room temperature (RT). The contribution of interface exchange coupling to the saturation of the FePt along the hard axis is then expected to be small.

XMCD measurements at the Fe $L_{2,3}$ edges were performed at the ID08 soft X-ray beamline of the European Synchrotron Radiation Facility (ESRF). XMCD spectra, acquired in a total electron yield mode (TEY), are obtained from the difference between several XAS spectra recorded by swapping the photon helicity with respect to the applied magnetic field. The spectral resolution is $E / \Delta E \sim 6000$ and the degree of circular polarization is close to $100 \%$. All spectra are corrected for TEY saturation effects [28] and are normalized far from the Fe $L_{2,3}$ edges (Fig. 3). Samples are allowed to rotate about an axis in the film plane, with the polar angle $\theta$ defined as the angle between the surface normal and the $\mathrm{x}$-ray propagation.

\section{RESULTS AND DISCUSSION}

As a first step we investigate the magnetic anisotropy of the samples. Element selective magnetization loops were obtained by measuring the maximum XMCD signal at the Fe $L_{3}$ edge [vertical line in Fig. 1(a)] as a function of the external magnetic field applied along the beam axis. For sample A the angular dependence of the magnetization loops at $10 \mathrm{~K}$ are shown in Fig. 2(a), where the sample is turned from $\theta=0^{\circ}$ (normal incidence) to $\theta=70^{\circ}$ (grazing incidence). The square-shaped loop at $\theta=0^{\circ}$ indicates that the magnetic anisotropy of the FePt layer is perpendicular to the surface. This PMA, with high remanence for $\theta=0^{\circ}$, persists above RT. Figure 2(b) shows the temperature dependence of the normalized magnetization at saturation, $M_{s}^{\mathrm{A}}(T) / M_{s}^{\mathrm{A}}(10)$, measured from the XMCD maximum at normal incidence and close to remanence. Based on Okamoto et al. [3], we use a Brillouin function with $J=10$ to adjust the data and estimate the Curie temperature $T_{C} \approx 450 \mathrm{~K}$. Such a reduction of $T_{C}$ with respect to the bulk value is to be expected since ferromagnetism is weakened as the thickness is reduced.

In order to estimate anisotropy constants, the approach to saturation of the hysteresis loops at $\theta=70^{\circ}$ has been adjusted in a coherent rotation picture, with the total energy density expressed by $E=K_{1} \sin ^{2} \gamma+K_{2} \sin ^{4} \gamma-\frac{\mu_{0} M_{s}^{2}}{2} \sin ^{2} \gamma-$ $\mu_{0} H M_{s} \cos (\theta-\gamma)$, where $\gamma$ is the angle between the magnetization and the normal (easy) axis. For the $10 \mathrm{~K}$ data [inset in Fig. 2(a), blue squares] we obtained (in units of $\left.\mathrm{MJ} / \mathrm{m}^{3}\right) K_{1}^{\mathrm{A}}(10)=3.5(5)$ and $K_{2}^{\mathrm{A}}(10)=0.6(3)$ (blue line), 


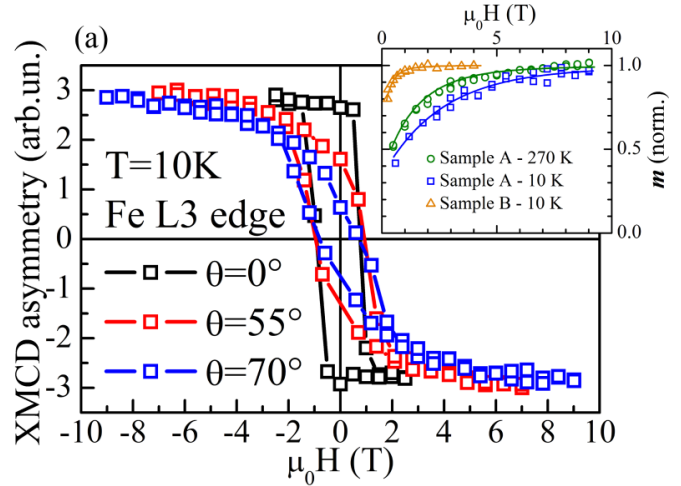

(b)

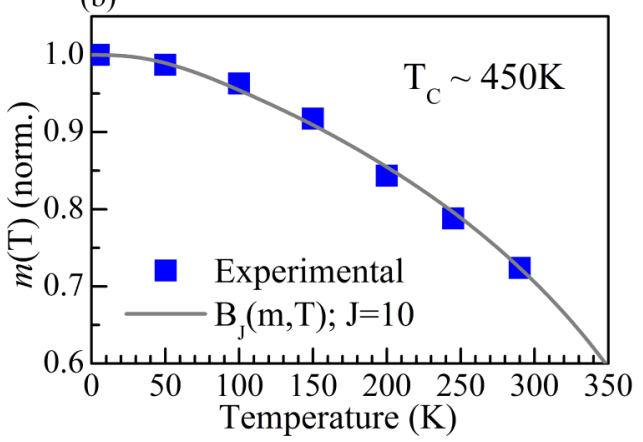

FIG. 2. (Color online) (a) Angular dependence of maximum Fe $L_{3} \mathrm{XMCD}$ as a function of the external magnetic field for sample A at $10 \mathrm{~K}$. The inset shows the approach to saturation for $\theta=70^{\circ}$ and the corresponding fit at 270 and $10 \mathrm{~K}$ and for sample B at $10 \mathrm{~K}$, as indicated in the legend. (b) Normalized magnetization at saturation vs temperature with a Brillouin fit $(J=10)$ for sample A.

with $M_{s}(10)=1.3 \times 10^{6} \mathrm{~A} / \mathrm{m}$ from Okamoto et al. [3]. The $K_{1}$ value is close to that observed by these authors [3] ( 4) for a $14 \mathrm{~nm}$ thick film with $S=0.72$ at $10 \mathrm{~K}$. To adjust the $270 \mathrm{~K}$ data (green circles), we took $M_{s}^{\mathrm{A}}(270)$ from Fig. 2(b) normalized by the value at $10 \mathrm{~K}, M_{s}(10)$, which gives $K_{1}^{\mathrm{A}}(270)=1.6(3)$ and $K_{2}^{\mathrm{A}}(270)=0.4(2)($ green line $)$. For this single point, the expected power law behavior [29] $K_{1}(T) / K_{1}(0) \approx\left[M_{S}(T) / M_{S}(0)\right]^{\alpha}$ would give an exponent $\alpha=2.8$, compatible with values reported for FePt, which range from $2[3,29]$ to 3 [30]. Such a high value of MAE for only $2-3 \mathrm{FePt}$ BL thickness is consistent with recent theoretical predictions [14].

A similar behavior has been observed for sample B, with high remanent magnetization [24] at $\theta=0^{\circ}$, evidencing PMA. At $\theta=70^{\circ}$ the approach to saturation of the hysteresis loops [inset in Fig. 2(a)] was adjusted with $K_{1}^{\mathrm{B}}(10)=1.1(2)$ and $K_{2}^{\mathrm{B}}(10)=0.2(1)$. The smaller MAE for sample B is consistent with the smaller order parameter. Systematic temperature dependent measurements are still missing for this sample, but the ratio of the XMCD signal at 300 and $10 \mathrm{~K}$ compares well to the expected temperature dependence of the magnetization for thick films [3], suggesting that $T_{C}$ is above the value found for sample $\mathrm{A}$, in agreement with its larger thickness.

We then turn to the determination of the Fe orbital $\left(m_{o}\right)$ and the effective spin $\left(m_{s}^{\text {eff }}\right)$ magnetic moments (Table I). $m_{s}^{\text {eff }}$ is defined by $m_{s}^{\text {eff }}=m_{s}+7 m_{T}$, where $m_{s}$ is the spin moment and $m_{T}$ the magnetic dipole term accounting for the asphericity of the spin density. From XMCD sum rules [19]
TABLE I. Fe magnetic moments (in $\mu_{B}$ ) for samples A and B and comparison with experimental (expt.) and theoretical (theor.) data. The available experimental data are obtained with $\theta=0^{\circ}$, apart from Ref. [21] with $\theta=30^{\circ}$ and Ref. [22], where the nanoparticles are randomly oriented. $m_{s}^{*}$ is $m_{s}^{\text {eff }}$ for experiments and $m_{s}$ for theory.

\begin{tabular}{lllll}
\hline \hline & $m_{s}^{*}$ & \multicolumn{1}{c}{$m_{o} \perp(/ \|)$} & $\Delta m_{o}$ & $m_{o} / m_{s}^{*} \perp(/ \|)$ \\
\hline A & & & & $0.04(1) / 0.03(1)$ \\
B & $2.7(3)$ & $0.11(1) / 0.08(1)$ & $0.03(2)$ & $0.040(3) / 0.031(3)$ \\
Expt. [20] & 2.40 & 0.21 & & 0.087 \\
Expt. [21] & 2.45 & 0.24 & & 0.10 \\
Expt. [22] & 2.38 & 0.20 & & 0.08 \\
Expt. [8] & 2.7 & & & 0.05 \\
Theor. [11] & 2.7 & 0.13 & 0.02 & 0.048 \\
Theor. [13] & 2.89 & $0.110 / 0.096$ & 0.014 & $0.038 / 0.033$ \\
Theor. [12] & 2.77 & 0.058 & 0.012 & 0.021 \\
Theor. [6] & & 0.088 & 0.004 & \\
Theor. [23] & 2.87 & 0.072 & & 0.025 \\
\hline \hline
\end{tabular}

$m_{o}=-4 n_{h} q / 3 r$ and $m_{s}^{\text {eff }}=-n_{h}(6 p-4 q) / r$, where $n_{h}$ is the theoretical number of $d$ holes, taken here as $n_{h}=3.4$ [22], $p=$ $\int_{L_{3}}\left(\mu_{+}-\mu_{-}\right) d E$, and $q=\int_{L_{3}+L_{2}}\left(\mu_{+}-\mu_{-}\right) d E$, as indicated in Fig. 3. The resonant intensity is given by the integral $r=\int_{L_{3}+L_{2}}\left(\mu_{+}+\mu_{-}\right) d E$, where a steplike background (gray line in Fig. 3) due to nonresonant excitation channels is subtracted. The ratio $m_{o} / m_{s}^{\text {eff }}=2 q /(9 p-6 q)$, independent of $r$ and $n_{h}$, is reported separately.

The critical issue for the quantitative determination of the individual values of $m_{o}$ and $m_{s}^{\text {eff }}$ is the accurate evaluation of the integral $q$, which strongly depends on the procedure of background subtraction in XMCD spectra. An improper background may result in overestimated values for the orbital moments. An uncorrected small vertical shift or a residual linear background in the XMCD spectrum results respectively in a linear or a parabolic deviation in its integral. In our data analysis procedure, we choose a linear background subtraction

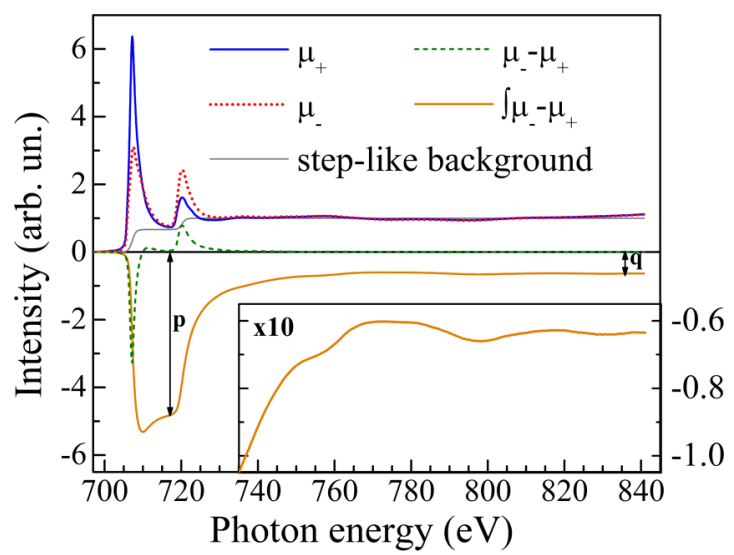

FIG. 3. (Color online) XAS, XMCD, and XMCD integral for sample $\mathrm{B}$, as indicated in the labels for $\theta=0^{\circ}$ and $T=10 \mathrm{~K}$. Also shown is the steplike function used as the background for an average XAS integral (charge sum rule, not shown). Inset: A ten times magnification of the XMCD integral evidences the oscillatory behavior coming from integration of the magnetic extended $\mathrm{x}$-ray absorption fine structure (EXAFS) oscillations, with convergence far above $800 \mathrm{eV}$. 
in order to ensure that the XMCD integral is zero before the $L_{3}$ edge $(\sim 700 \mathrm{eV})$ and that it converges far above the $L_{2}$ edge $(\sim 840 \mathrm{eV})$ (inset of Fig. 3). Such a convergence test over a broad energy range is required for a proper background subtraction and to obtain reliable values of the orbital moments.

The test of the convergence of the XMCD integrals, which requires acquisition of broad energy spectra, is more sensitive to any background and is required when looking for small differences in the orbital moments as functions of the x-ray incidence. The spectra in Fig. 3 were obtained at $10 \mathrm{~K}$, applying along the beam axis an external field (4 T) that is large enough to saturate the sample, whatever the orientation. With the above analysis procedure we obtained at $\theta=0^{\circ}(\perp)$ and $\theta=70^{\circ}(\|)$, the values reported in Table I. The error bars are estimated by the standard deviation after averaging several measurements with opposite external fields. One should note the large OMA, $\Delta m_{o}^{\mathrm{B}}=m_{o}^{\mathrm{B}}(\perp)-m_{o}^{\mathrm{B}}(\|)=0.03(2) \mu_{B}$.

For sample $A$, the presence of the interface Fe oxide prevents us from determining the absolute values for the magnetic moments, since the oxide contributes to the XAS but not to the XMCD spectra (Fig. 1) and causes an overestimation of the integral $r$. However, the ratio $m_{o} / m_{s}^{\text {eff }}$ can still be determined and is reported for both orientations (Table I), measured at $270 \mathrm{~K}$ and with an external field of $9 \mathrm{~T}$. The presence of a Co $L_{3}$ edge $(\sim 780 \mathrm{eV})$ limits the integration range to about $50 \mathrm{eV}$ above the Fe $L_{2}$ edge. This precludes any confirmation of the convergence of the XMCD integral far above the Fe edge, resulting in larger error bars for the magnetic moments. An average of two XMCD spectra acquired with opposite magnetic fields is used to limit systematic errors.

It is noteworthy that in both samples $m_{o} / m_{s}^{\text {eff }}(\perp)$ is equal to 0.04 . This value approximates quite well the ratio $m_{o}^{\perp} / m_{s}$ obtained by ab initio calculations [6,11-13,23] (0.021-0.048) (Table I). We should note that the calculations of Ravindran et al. [13] and Daalderop et al. [11] (Table I) take into account the orbital polarization correction to include the effects of Hund's second rule. Their values for $m_{o}, \Delta m_{o}$, and $m_{o} / m_{s}$ are in the closest agreement with the experimental values obtained in the present work. The agreement still holds if we take into account the dipolar correction $\left(7 m_{T}\right)$ to $m_{s}^{\text {eff }}$, of the order of $2 \%$ [19] to $20 \%$ [23]. We should note that this $m_{o} / m_{s}^{\text {eff }}(\perp)$ ratio is smaller by a factor around 2 with respect to most of the experimental ratios reported in the literature for FePt [20-22] and slightly smaller than the value reported by Imada et al. [8] (Table I).

The experimental values previously reported were given for nanoparticles [22] or for thicker films with similar [8,21] or higher [20] order parameters. This could explain part of the discrepancy with our experiments. However, we claim that the difference is likely to have its origin in systematic errors. The presence of a background in the XMCD spectrum or a reduced integration range are possible sources of systematic errors for quantifying $q$. This is the case of Soares et al. [10], where a reevaluation of the data showed the presence of a nonlinear background that overestimated $m_{o}$. In the case of $\mathrm{Xu}$ et al. [20], a reduced integration range (up to $735 \mathrm{eV}$ ) may cause an overestimation of $m_{o} / m_{s}^{\text {eff }}$ by $65 \%$, according to the inset of Fig. 3. This last correction could reconcile the results of Imada et al. [8] and Xu et al. [20] with our own measurements.
The significant anisotropy of the Fe orbital moment observed $\left(\Delta m_{o}=0.03 \mu_{B}\right)$ is of the same order, even if slightly higher, as the few theoretical predictions available (Table I). We should also note that the calculations of Ravindran et al. [13] and Daalderop et al. [11] (Table I) take into account the orbital polarization correction to include the effects of Hund's second rule. Their values for $m_{o}, \Delta m_{o}$, and $m_{o} / m_{s}$ are in closest agreement with the experimental values obtained in the present work. An intense theoretical research effort has been dedicated to the study of MAE in FePt and other transition metal/noble metal alloys using different approaches [5-7,11-15,21]. It is a common statement that the key for the large MAE [5,6,11,12] is the combination of the large Pt SOI and the hybridization of Pt $5 d$ orbitals with $\mathrm{Fe}$ $3 d$ orbitals (highly spin polarized). If the Pt SOI is switched off [5] or reduced [11], the calculated MAE drops drastically, evidencing its major importance. The different approaches also agree that there is an increase of the MAE with an increase of the $c / a$ ratio $[5,7,14,15]$. This feature is attributed to an increase of hybridization between $\mathrm{Fe}$ and $\mathrm{Pt}$ with an decrease of the in-plane lattice parameter and subsequent changes in the band filling of Fe $3 d$ orbitals [15]. Our highly distorted epitaxial layers $(c / a \sim 0.91)$ would, according to theory, have a smaller anisotropy than if they were fully relaxed or under a compressive strain. However, the $c / a$ dependence of anisotropy is irrelevant compared to the dependence with the order parameter [7].

Beyond these agreements, the orbital magnetism of both $\mathrm{Fe}$ and $\mathrm{Pt}$ atoms and its relationship with the origin of MAE in FePt has been the object of intense theoretical debate. The proportionality between the OMA and MAE [16], $\Delta E=\frac{\xi_{\text {eff }}}{4 \mu_{B}} \Delta m_{o}$, with $\xi_{\text {eff }}$ an effective coefficient of SOI, is known as the Bruno's model. Such a model was questioned for binary $3 d-5 d$ alloys [17]. Solovyev et al. [12] state that the MAE in FePt and CoPt alloys is entirely due to the Pt sublattice. The role of $\mathrm{Fe}$ and $\mathrm{Co}$ atoms would be only to induce exchange splitting in Pt via orbital hybridization. In this picture, followed by Kota et al. [6], the anisotropy of the $\mathrm{Pt}$ orbital moment is dominant upon Fe OMA. Moreover, the $\mathrm{Pt}$ orbital moment is larger in the direction perpendicular to the $c$ axis $[6,13]$ and that would give a negative $\xi_{\text {eff }}$.

On the other hand, Burkert et al. [5] claim that about $70 \%$ of the total MAE originates from the $\mathrm{Fe}$ atom. In the same way, Ravindran et al. [13] conclude that the magnetic anisotropy is mainly originating from Fe atoms. Zhu et al. [15] found a nearly linear relation between MAE and Fe OMA by changing the $c / a$ ratio, in agreement with Bruno's model. They deduce a positive $\xi_{\text {eff }}$ by only taking into account the Fe contribution. An enhanced Fe OMA would originate from the strong hybridization with the large SOI Pt atom [5,31]. Using the theoretical SOI coefficient of Fe [6] $\left(\xi_{\mathrm{Fe}} \sim 50 \mathrm{meV}\right)$ and the measured OMA $\left(\Delta m_{o}=0.03 \mu_{B}\right)$ in Bruno's model, we obtain $\Delta E \approx 2 \mathrm{MJ} / \mathrm{m}^{3}$. This value is compatible with the anisotropy constants reported here for sample B.

Our experimental result supports then the scenario where the hybridization-induced enhancement of Fe OMA dominates the large MAE, in agreement with Bruno's model for Fe. In our understanding, Fe satisfies the requirement of a large exchange splitting and narrow bandwidth for application of 
this model, while Pt shows a rather small exchange splitting while having a widespread density of states [13] that prevents one from using this perturbation treatment. The investigation of Pt magnetic moments for our samples, which would fairly complete this study, is precluded here by the presence of $\mathrm{Pt}$ as the substrate and cap layer. We should also point out that our FePt layers have an interface with an antiferromagnetic material. An influence of such an interface on the orbital magnetism cannot be fully discarded, even if it is expected that its effect on the strong PMA of FePt would be minor and vanish above room temperature [24,25].

\section{CONCLUSION}

In conclusion, we have investigated the anisotropy of the Fe orbital magnetic moment in epitaxial ultrathin $(<2 \mathrm{~nm}) \mathrm{FePt}$ layers by angular dependent $\mathrm{X}$-ray magnetic circular dichroism (XMCD) at the Fe $L$ edges for two distinct epitaxial systems
$\mathrm{CoO} / \mathrm{FePt} / \mathrm{Pt}(001)$ and $\mathrm{Pt} / \mathrm{FePt} / \mathrm{MnPt} / \mathrm{Pt}(001)$. We have found anisotropy constants consistent with the corresponding chemical order parameter. The Fe orbital magnetic moment has been deduced from a procedure for XMCD sum rule analysis with integration over a large energy range, reducing background errors. Our magnetic orbital moment values are found to be lower than most of the experimental values reported in the literature, but quite comparable to the theoretical ones. Moreover, we have observed a significant OMA of $0.03 \mu_{B}$. This OMA is discussed in light of the current theoretical understanding of magnetocrystalline anisotropy in ordered alloys.

\section{ACKNOWLEDGMENTS}

We would like to thank N. Brookes for fruitful discussions. Beamtime is acknowledged at the ID08 beamline of European Synchrotron Radiation Facility.
[1] S. Mangin, D. Ravelosona, J. Katine, M. Carey, B. Terris, and E. Fullerton, Nat. Mater. 5, 210 (2006).

[2] M. H. Kryder, E. C. Gage, T. W. Mcdaniel, W. A. Challener, R. E. Rottmayer, G. Ju, Y.-T. Hsia, and M. F. Erden, Proc. IEEE 96, 1810 (2008).

[3] S. Okamoto, N. Kikuchi, O. Kitakami, T. Miyazaki, Y. Shimada, and K. Fukamichi, Phys. Rev. B 66, 024413 (2002).

[4] A. Hotta, T. Ono, M. Hatayama, K. Tsumura, N. Kikuchi, S. Okamoto, O. Kitakami, and T. Shimatsu, J. Appl. Phys. 115, 17B712 (2014).

[5] T. Burkert, O. Eriksson, S. I. Simak, A. V. Ruban, B. Sanyal, L. Nordström, and J. M. Wills, Phys. Rev. B 71, 134411 (2005).

[6] Y. Kota and A. Sakuma, J. Phys. Soc. Jpn. 81, 084705 (2012).

[7] Y. Kota and A. Sakuma, J. Appl. Phys. 111, 07A310 (2012).

[8] S. Imada, A. Yamasaki, S. Suga, T. Shima, and K. Takanashi, Appl. Phys. Lett. 90, 132507 (2007).

[9] K. He, L. J. Zhang, X. C. Ma, J. F. Jia, Q. K. Xue, and Z. Q. Qiu, Phys. Rev. B 72, 155432 (2005).

[10] M. M. Soares, H. C. N. Tolentino, M. De Santis, A. Y. Ramos, and J. C. Cezar, J. Appl. Phys. 109, 07D725 (2011).

[11] G. H. O. Daalderop, P. J. Kelly, and M. F. H. Schuurmans, Phys. Rev. B 44, 12054 (1991).

[12] I. V. Solovyev, P. H. Dederichs, and I. Mertig, Phys. Rev. B 52, 13419 (1995).

[13] P. Ravindran, A. Kjekshus, H. Fjellvåg, P. James, L. Nordström, B. Johansson, and O. Eriksson, Phys. Rev. B 63, 144409 (2001).

[14] R. V. Chepulskii and W. H. Butler, Appl. Phys. Lett. 100, 142405 (2012).

[15] W. Zhu, H.-C. Ding, S.-J. Gong, Y. Liu, and C.-G. Duan, J. Phys.: Condens. Matter 25, 396001 (2013).

[16] P. Bruno, Phys. Rev. B 39, 865 (1989).

[17] C. Andersson, B. Sanyal, O. Eriksson, L. Nordström, O. Karis, D. Arvanitis, T. Konishi, E. Holub-Krappe, and J. H. Dunn, Phys. Rev. Lett. 99, 177207 (2007).
[18] B. T. Thole, P. Carra, F. Sette, and G. van der Laan, Phys. Rev. Lett. 68, 1943 (1992).

[19] C. T. Chen, Y. U. Idzerda, H.-J. Lin, N. V. Smith, G. Meigs, E. Chaban, G. H. Ho, E. Pellegrin, and F. Sette, Phys. Rev. Lett. 75, 152 (1995).

[20] D. B. Xu, C. J. Sun, J. S. Chen, S.-W. Han, S. M. Heald, R. A. Rosenberg, and G. M. Chow, J. Appl. Phys. 111, 07C120 (2012).

[21] C. J. Sun, G. M. Chow, G. H. Fecher, J. S. Chen, H.-J. Lin, and Y. Hwu, Jpn. J. Appl. Phys. 45, 2539 (2006).

[22] C. Antoniak, M. E. Gruner, M. Spasova, A. V. Trunova, F. M. Römer, A. Warland, B. Krumme, K. Fauth, S. Sun, P. Entel, M. Farle, and H. Wende, Nat. Commun. 2, 528 (2011).

[23] I. Galanakis, M. Alouani, and H. Dreyssé, J. Magn. Magn. Mater. 242-245, 27 (2002).

[24] M. M. Soares, M. De Santis, H. C. N. Tolentino, A. Y. Ramos, M. El Jawad, Y. Gauthier, F. Yildiz, and M. Przybylski, Phys. Rev. B 85, 205417 (2012).

[25] A. D. Lamirand, M. M. Soares, A. Y. Ramos, H. C. N. Tolentino, M. De Santis, J. C. Cezar, A. de Siervo, and M. Jamet, Phys. Rev. B 88, 140401 (2013).

[26] A. D. Lamirand, M. D. Santis, M. M. Soares, A. Y. Ramos, S. Grenier, and H. C. N. Tolentino, arXiv:1410.6833 [J. Phys.: Condens. Matter (to be published)].

[27] A. D. Lamirand, M. M. Soares, A. Y. Ramos, H. C. Tolentino, M. De Santis, J. C. Cezar, and A. de Siervo, J. Magn. Magn. Mater. 373, 6 (2015).

[28] R. Nakajima, J. Stöhr, and Y. U. Idzerda, Phys. Rev. B 59, 6421 (1999).

[29] J. B. Staunton, S. Ostanin, S. S. A. Razee, B. L. Gyorffy, L. Szunyogh, B. Ginatempo, and E. Bruno, Phys. Rev. Lett. 93, 257204 (2004)

[30] T. Bublat and D. Goll, J. Appl. Phys. 108, 113910 (2010).

[31] I. Galanakis, P. M. Oppeneer, P. Ravindran, L. Nordström, P. James, M. Alouani, H. Dreyssé, and O. Eriksson, Phys. Rev. B 63, 172405 (2001). 